PROCEEDINGS OF THE

AMERICAN MATHEMATICAL SOCIETY

Volume 135, Number 1, January 2007, Pages 243-251

S 0002-9939(06)08455-3

Article electronically published on June 30, 2006

\title{
CONTINUITY OF THE MAXIMAL OPERATOR IN SOBOLEV SPACES
}

\author{
HANNES LUIRO
}

(Communicated by Juha M. Heinonen)

\begin{abstract}
We establish the continuity of the Hardy-Littlewood maximal operator on Sobolev spaces $W^{1, p}\left(\mathbb{R}^{n}\right), 1<p<\infty$. As an auxiliary tool we prove an explicit formula for the derivative of the maximal function.
\end{abstract}

\section{INTRODUCTION}

The classical Hardy-Littlewood maximal operator $\mathcal{M}$ is defined on $L_{\text {loc }}^{1}\left(\mathbb{R}^{n}\right)$ by setting for all $f \in L_{l o c}^{1}\left(\mathbb{R}^{n}\right)$

$$
\mathcal{M} f(x)=\sup _{r>0} f_{B(x, r)}|f(y)| d y=\sup _{r>0} \frac{1}{m\left(B_{r}\right)} \int_{B(x, r)}|f(y)| d y,
$$

for every $x \in \mathbb{R}^{n}$; here $m$ denotes the Lebesgue measure in $\mathbb{R}^{n}$ and $B_{r}=B(0, r)$.

The theorem of Hardy, Littlewood and Wiener asserts that $\mathcal{M}$ is bounded on $L^{p}\left(\mathbb{R}^{n}\right)$ for $1<p \leq \infty$. This theorem is one of the cornerstones of harmonic analysis. Applications e.g. to the study of Sobolev-functions indicate that it is also useful to know how it preserves differentiability properties of functions. Quite recently, Kinnunen observed $[\mathrm{K}]$ that $\mathcal{M}$ is bounded on the Sobolev-space $W^{1, p}\left(\mathbb{R}^{n}\right)$, for $1<p \leq \infty$. Extensions and related results can be found from e.g. $\mathrm{KL}$, $\mathrm{KO}$, $\mathrm{KS}$, [HO].

Continuity of the maximal operator in $L^{p}\left(\mathbb{R}^{n}\right)$ follows from its sublinearity and boundedness. Because of boundedness in $W^{1, p}\left(\mathbb{R}^{n}\right)$, it is very natural to ask whether the maximal operator is continuous in $W^{1, p}\left(\mathbb{R}^{n}\right), 1<p<\infty$, or not. This question was posed in [HO, Question 3] where it was attributed to T. Iwaniec. In general, bounded non-sublinear operators need not be continuous. An important example of this kind of phenomenon is the result of Almgren and Lieb $\mathrm{AL}$ who proved that the (known to be bounded) symmetric rearrangement $\mathcal{R}: W^{1, p}\left(\mathbb{R}^{n}\right) \mapsto W^{1, p}\left(\mathbb{R}^{n}\right)$ is not continuous when $1<p<n$ and $n>1$. On Sobolev-spaces, $\mathcal{M}$ is not sublinear and the issue of the continuity of $\mathcal{M}$ is not trivial even though we know the boundedness.

Our main result (Theorem 4.1 below) is the positive answer to the question of Iwaniec. A central role in our proof is played by a careful analysis of the set $\mathcal{R} f(x)$

Received by the editors June 4, 2004 and, in revised form, August 8, 2005.

2000 Mathematics Subject Classification. Primary 42B25, 46E35, 47H99.

Key words and phrases. Maximal function, Sobolev spaces, continuity, regularity.

The author was supported by the Academy of Finland, project 201015.

(C)2006 American Mathematical Society Reverts to public domain 28 years from publication 
(see 2.1 below), which consists of the radii $r$ for which equality is achieved in (1). As a useful auxiliary tool we establish in Theorem 3.1 an explicit formula for the derivative of the maximal function.

\section{Definitions And AUXiliary RESUlts}

Let us first introduce some notation. If $A \subset \mathbb{R}^{n}$ and $r \in \mathbb{R}^{n}$, we define

$$
d(r, A):=\inf _{a \in A}|r-a| \text {, and } A_{(\lambda)}:=\left\{x \in \mathbb{R}^{n}: d(x, A) \leq \lambda\right\} \text { for } \lambda \geq 0 .
$$

We endow $W^{1, p}\left(\mathbb{R}^{n}\right)$ with the norm

$$
\|f\|_{1, p}=\|f\|_{p}+\|\nabla f\|_{p}
$$

where $\nabla f$ is the weak gradient of $f$. Let us also denote by $\|f\|_{p, A}$ the $L^{p}$-norm of $\chi_{A} f$ for all measurable sets $A \subset \mathbb{R}^{n}$.

The following new concept will be central in this work.

Definition 2.1. Let $f \in L^{p}\left(\mathbb{R}^{n}\right), 1 \leq p<\infty$. The set $\mathcal{R} f(x)$ is defined as

$$
\mathcal{R} f(x)=\left\{r \geq 0: M f(x)=\limsup _{r_{k} \rightarrow r} f_{B\left(x, r_{k}\right)}|f(y)| d y \text {, for some } r_{k}>0\right\} .
$$

Remarks. We comment on the above definition and the properties of the sets $\mathcal{R} f(x)$. First, the definition clearly implies that $\mathcal{R} f(x)$ is always closed. Moreover, for fixed $x \in \mathbb{R}^{n}$ define $u_{x}:[0, \infty) \mapsto \mathbb{R}$ by

$$
u_{x}(0)=|f(x)| \text { and } u_{x}(r)=f_{B(x, r)}|f(y)| d y \text { when } r \in(0, \infty) .
$$

First of all, the functions $u_{x}$ are continuous for almost all $x$. The continuity on $(0, \infty)$ is clearly true for all $x$ and at 0 it follows a.e., because almost every point $x \in \mathbb{R}^{n}$ is a Lebesgue point for $f$. Moreover, by Hölder's inequality we have

$$
u_{x}(r) \leq\|f\|_{p}\left(m\left(B_{r}\right)\right)^{\frac{1}{q}-1},
$$

where $q$ is the conjugate exponent of $p$, and hence $\lim _{r \rightarrow \infty} u_{x}(r)=0$.

These facts together imply that, for almost all $x$, the function $u_{x}$ has at least one maximum point in $[0, \infty)$. Furthermore, they guarantee that for all $x \in \mathbb{R}^{n}$ the set $\mathcal{R} f(x)$ is nonempty and

$$
\begin{aligned}
& M f(x)=f_{B(x, r)}|f(y)| d y \text { if } r \in \mathcal{R} f(x) \text { and } r>0, \forall x \in \mathbb{R}^{n}, \text { and } \\
& M f(x)=|f(x)| \text { for almost every } x \text { such that } 0 \in \mathcal{R} f(x) .
\end{aligned}
$$

Also, it is useful to observe that for every $R>0$ (assuming $f \not \equiv 0$ ) it is true that

$$
\sup \{r: r \in \mathcal{R} f(x), x \in B(0, R)\}<\infty .
$$

The following lemma tells us how the sets $\mathcal{R} f(x)$ and $\mathcal{R} g(x)$ are related to each other, especially when $\|f-g\|_{p}$ is small.

Lemma 2.2. Let $1 \leq p<\infty$ and suppose $f_{j} \rightarrow f$ in $L^{p}\left(\mathbb{R}^{n}\right)$ when $j \rightarrow \infty$. Then for all $R>0$ and $\lambda>0$ it holds that

$$
m\left(\left\{x \in B(0, R): \mathcal{R} f_{j}(x) \not \subset \mathcal{R} f(x)_{(\lambda)}\right\}\right) \rightarrow 0 \text { if } j \rightarrow \infty .
$$


Proof. First we indicate why the above set is always Lebesgue-measurable when $f$ and all the functions $f_{j}$ are in $L^{p}\left(\mathbb{R}^{n}\right)$. The continuity of the average functions $u_{x}$, for almost every $x$, is used as a main tool in the following argument. Let the set $\mathcal{N}$ consist of those points which are not Lebesgue points of any of the functions $f_{j}$ or $f$, especially, $m(\mathcal{N})=0$. Moreover we denote by $Q_{+}$the set of positive rationals. Now we can write

$$
\begin{aligned}
& \left\{x: \mathcal{R} f_{j}(x) \not \subset \mathcal{R} f(x)_{\lambda}\right\} \backslash \mathcal{N} \\
& =\bigcup_{i=1}^{\infty} \bigcap_{m=1}^{\infty}\left\{x: \exists r>0 \text { s.t. } d(r, \mathcal{R} f(x))>\lambda+\frac{1}{i} \text { and } M f_{j}(x)<f_{B(x, r)} f_{j}+\frac{1}{m}\right\} \\
& =\bigcup_{i=1}^{\infty} \bigcap_{m=1}^{\infty} \bigcup_{q \in \mathcal{Q}_{+}}\left[\left\{x: d(q, \mathcal{R} f(x))>\lambda+\frac{1}{i}\right\} \cap\left\{x: M f_{j}(x)<f_{B(x, q)} f_{j}+\frac{1}{m}\right\}\right] .
\end{aligned}
$$

From this we conclude that it is enough to prove that the set $\{x: d(q, \mathcal{R} f(x))>\lambda\}$ is measurable for arbitrary $q$ and $\lambda$. Using the same reasoning as above, especially the continuity of the expression $\underset{B(x, r)}{f}|f|$ as a function of $r$, we write that

$$
\{x: d(q, \mathcal{R} f(x))>\lambda\}=\bigcup_{k=1}^{\infty} \bigcap_{q^{\prime} \in Q_{+} \cap[q-\lambda, q+\lambda]}\{x: M f(x)>\underbrace{}_{B\left(x, q^{\prime}\right)} f+\frac{1}{k}\} .
$$

This implies the measurability.

Then we are ready to prove the lemma. It is sufficient to prove the claim in the case where both $f$ and $f_{j}$ are nonnegative, because $\mathcal{R} f(x)=\mathcal{R}|f|(x)$. Observe that $\mathcal{R} f(x)$ is $[0, \infty)$ for all $x$ if $f \equiv 0$ a.e., whence this case is trivial. Let $\lambda>0, R>0$ and $\varepsilon>0$. For almost every $x \in B(0, R)$ there exists a natural number $i(x) \in \mathbb{N}$ so that

$$
f_{B(x, r)} f(y) d y<M f(x)-\frac{1}{i(x)}, \text { when } d(r, \mathcal{R} f(x))>\lambda .
$$

This can be seen in the following way: If the claim is not true there is a sequence of radii $\left(r_{k}\right)_{k=1}^{\infty}$ so that

$$
f_{B\left(x, r_{k}\right)} f(y) d y \rightarrow M f(x) \text { and } d\left(r_{k}, \mathcal{R} f(x)\right)>\lambda .
$$

By moving to a subsequence, if needed, we may assume that $r_{k} \rightarrow r$ as $k \rightarrow \infty$, because (2) implies that the sequence $\left(r_{k}\right)_{k=1}^{\infty}$ must be bounded. It follows that $r \in \mathcal{R} f(x)$. This is a contradiction, since obviously $r$ satisfies $d(r, \mathcal{R} f(x)) \geq \lambda$.

From (3) we conclude that there exists $i \in \mathbb{N}$ so that

$$
B(0, R) \subset\left\{x: f_{B(x, r)} f(y) d y<M f(x)-\frac{1}{i}, \text { if } d(r, \mathcal{R} f(x))>\lambda\right\} \cup E=: A \cup E,
$$

where $E$ is a measurable set with $m(E)<\varepsilon$. The weak type $(1,1)$-estimate for the maximal operator implies that there exists $j_{0} \in \mathbb{N}$ so that

$$
m\left(\left\{x \in B(0, R):\left|M\left(f-f_{j}\right)(x)\right| \geq \frac{1}{4 i}\right\}\right)<\varepsilon \text { when } j \geq j_{0} .
$$


For all $j$ we observe that

$$
\begin{aligned}
A \subset & \left\{x: f_{B(x, r)} f_{j}(y) d y<M f(x)-\frac{1}{2 i}, \text { if } d(r, \mathcal{R} f(x))>\lambda\right\} \\
& \cup\left\{x:\left|f_{B(x, r)} f(y) d y-f_{B(x, r)} f_{j}(y) d y\right| \geq \frac{1}{2 i}, \text { for some } r, d(r, \mathcal{R} f(x))>\lambda\right\} \\
=: & A_{j} \cup B_{j} .
\end{aligned}
$$

Continuing the same reasoning, and using the fact that $\left|M f(x)-M f_{j}(x)\right| \leq$ $\left|M\left(f-f_{j}\right)(x)\right|$, we get

$$
\begin{aligned}
& A_{j} \subset\left\{x: f_{B(x, r)} f_{j}(y) d y<M f_{j}(x)-\frac{1}{4 i}, \text { if } d(r, \mathcal{R} f(x))>\lambda\right\} \\
& \cup\left\{x:\left|M\left(f-f_{j}\right)(x)\right| \geq \frac{1}{4 i}\right\} \\
&=: C_{j} \cup D_{j} .
\end{aligned}
$$

Now

$$
C_{j} \subset\left\{x: \mathcal{R} f_{j}(x) \subset \mathcal{R} f(x)_{(\lambda)}\right\} .
$$

By combining the above observations, we conclude that for all $j$

$$
B(0, R) \subset\left\{x: \mathcal{R} f_{j}(x) \subset \mathcal{R} f(x)_{(\lambda)}\right\} \cup E \cup D_{j} \cup B_{j} .
$$

Observe finally that $B_{j} \subset D_{j}$ and, by our choice of $j_{0}$ we have $m\left(D_{j}\right)<\varepsilon$ if $j \geq j_{0}$, and therefore

$$
m\left(\left\{x \in B(0, R): \mathcal{R} f_{j}(x) \not \subset \mathcal{R} f(x)_{(\lambda)}\right\}\right)<2 \varepsilon,
$$

if $j \geq j_{0}$.

Let us introduce more notation. Assume that $f \in L^{p}\left(\mathbb{R}^{n}\right), 1 \leq p<\infty$. Let $e_{i}$ be one of the standard basevectors of $\mathbb{R}^{n}$. For all $h \in \mathbb{R},|h|>0$, we define the functions $f_{h}^{i}$ and $f_{\tau(h)}^{i}$ by setting

$$
f_{h}^{i}(x)=\frac{f\left(x+h e_{i}\right)-f(x)}{|h|} \text { and } f_{\tau(h)}^{i}(x)=f\left(x+h e_{i}\right) .
$$

Now we know that $f_{\tau(h)}^{i} \rightarrow f$ in $L^{p}\left(\mathbb{R}^{n}\right)$ when $|h| \rightarrow 0$ and, if $p>1$, for functions $f \in W^{1, p}\left(\mathbb{R}^{n}\right)$ we have (see [GT, 7.11]) that $f_{h}^{i} \rightarrow D_{i} f$ in $L^{p}\left(\mathbb{R}^{n}\right)$ when $|h| \rightarrow 0$.

Corollary 2.3. Let $f \in L^{p}\left(\mathbb{R}^{n}\right), 1 \leq p<\infty$. Then for all $i, 1 \leq i \leq n, R>$ $0, \lambda>0$ one has

$$
m\left(\left\{x \in B(0, R): \mathcal{R} f(x) \not \subset \mathcal{R} f_{\tau(h)}^{i}(x)_{(\lambda)} \text { or } \mathcal{R} f_{\tau(h)}^{i}(x) \not \subset \mathcal{R} f(x)_{(\lambda)}\right\}\right) \stackrel{h \rightarrow 0}{\longrightarrow} 0 .
$$

Proof. Now $f_{\tau(h)}^{i} \rightarrow f$ and as a consequence of Lemma 2.2 it is clearly sufficient to prove that

$$
m\left(\left\{x \in B_{R}: \mathcal{R} f(x) \not \subset \mathcal{R} f_{\tau(h)}^{i}(x)_{(\lambda)}\right\}\right) \rightarrow 0 \text { as } h \rightarrow 0 .
$$

But this also follows easily from Lemma 2.2. because for $|h|<1$ one has that

$$
\begin{aligned}
& \left\{x \in B_{R}: \mathcal{R} f(x) \not \subset \mathcal{R} f_{\tau(h)}^{i}(x)_{(\lambda)}\right\} \\
= & \left\{x \in B_{R}: \mathcal{R} f_{\tau(-h)}^{i}\left(x+h e_{i}\right) \not \subset \mathcal{R} f\left(x+h e_{i}\right)_{(\lambda)}\right\} \\
\subset & \left\{y \in B_{R+1}: \mathcal{R} f_{\tau(-h)}^{i}(y) \not \subset \mathcal{R} f(y)_{(\lambda)}\right\}-h e_{i} .
\end{aligned}
$$


Remark. The previous corollary will became useful after the following observation. Let us denote by

$$
\pi(A, B):=\inf \left\{\delta>0: A \subset B_{(\delta)} \text { and } B \subset A_{(\delta)}\right\}
$$

the Hausdorff distance of the sets $A$ and $B$. Let $f$ be in $L^{p}\left(\mathbb{R}^{n}\right)$. With the new notation, the corollary says that

$$
m\left(\left\{x \in B_{R}: \pi\left(\mathcal{R} f(x), \mathcal{R} f\left(x+h e_{i}\right)\right)>\lambda\right\}\right) \rightarrow 0 \text { when } h \rightarrow 0 .
$$

Therefore we easily infer that there is a sequence $\left(h_{k}\right)_{k=1}^{\infty}, h_{k}>0$ with $h_{k} \rightarrow 0$, and such that $\pi\left(\mathcal{R} f(x), \mathcal{R} f\left(x+h_{k} e_{i}\right)\right) \rightarrow 0$ as $k \rightarrow \infty$ for almost every $x \in B_{R}$. This is the decisive fact needed in the following section.

\section{A FORMUla FOR the DERIVATIVE OF the MAXimal FUnCtion}

Let us denote by $D_{i} f(x)$ the partial derivative $\frac{\partial f}{\partial x_{i}}$.

Theorem 3.1. Let $f \in W^{1, p}\left(\mathbb{R}^{n}\right), 1<p<\infty$. Then we have for almost all $x \in \mathbb{R}^{n}$ that

(1) $D_{i} M f(x)=f_{B(x, r)} D_{i}|f|(y) d y$ for all $r \in \mathcal{R} f(x), r>0$, and

(2) $D_{i} M f(x)=D_{i}|f|(x)$ if $0 \in \mathcal{R} f(x)$.

Proof. It is sufficient to prove the claim for nonnegative functions, because $M f=$ $M|f|$ and $|f| \in W^{1, p}\left(\mathbb{R}^{n}\right)$ if $f \in W^{1, p}\left(\mathbb{R}^{n}\right)$. Let $R>0$. We start by choosing a sequence $\left(h_{k}\right)_{k=1}^{\infty}, h_{k}>0$ and $h_{k} \rightarrow 0$, so that $\pi\left(\mathcal{R} f(x), \mathcal{R} f\left(x+h_{k} e_{i}\right)\right) \rightarrow 0$ as $k \rightarrow \infty$ for almost all $x \in B_{R}$ (see the Remark after Corollary 2.3). Then we have

$$
\begin{aligned}
& \left\|D_{i} M f-(M f)_{h_{k}}^{i}\right\|_{p, B_{R}} \rightarrow 0 \text { as } k \rightarrow \infty, \\
& \left\|D_{i} f-f_{h_{k}}^{i}\right\|_{p, B_{R}} \rightarrow 0 \text { as } k \rightarrow \infty, \\
& \left\|M\left(D_{i} f-f_{h_{k}}^{i}\right)\right\|_{p, B_{R}} \rightarrow 0 \text { as } k \rightarrow \infty .
\end{aligned}
$$

Now, by extracting a subsequence if needed, we may assume that the convergences above are true pointwise almost everywhere as well. Moreover, we recall that the set

$$
\left\{x \in \mathbb{R}^{n}: \exists k \in \mathbb{N} \text { s.t. } 0 \in \mathcal{R} f\left(x+h_{k} e_{i}\right) \text { with } M f\left(x+h_{k} e_{i}\right) \neq f\left(x+h_{k} e_{i}\right)\right\}
$$

has measure zero as a countable union of the sets having measure zero. Let $x \in B_{R}$ be a Lebesgue point of both $f$ and $D_{i} f$ outside the union of all these unwanted sets of measure zero (in particular, the pointwise analogies of (i)-(iii) hold at $x$ ) and let $r \in \mathcal{R} f(x)$. 
Now, because $\pi\left(\mathcal{R} f(x), \mathcal{R} f\left(x+h_{k} e_{i}\right)\right) \rightarrow 0$, we find radii $r_{k} \in \mathcal{R} f\left(x+h_{k} e_{i}\right)$ so that $r_{k} \rightarrow r$ when $k \rightarrow \infty$. If $r>0$ we can estimate:

$$
\begin{aligned}
D_{i} M f(x) & =\lim _{k \rightarrow \infty} \frac{1}{h_{k}}\left(M f\left(x+h_{k} e_{i}\right)-M f(x)\right) \\
& \leq \lim _{k \rightarrow \infty} \frac{1}{h_{k}}\left(f_{B\left(x+h_{k} e_{i}, r_{k}\right)} f(y) d y-f_{B\left(x, r_{k}\right)} f(y) d y\right) \\
& =\lim _{k \rightarrow \infty} \frac{1}{m\left(B\left(x, r_{k}\right)\right)} \int_{B\left(x, r_{k}\right)} \frac{f\left(y+h_{k} e_{i}\right)-f(y)}{h_{k}} d y \\
& =f_{B(x, r)} D_{i} f(y) d y .
\end{aligned}
$$

The last equation holds, because $m\left(B_{r_{k}}\right) \rightarrow m\left(B_{r}\right)$ and

$$
\chi_{B\left(x, r_{k}\right)} f_{h_{k}}^{i} \rightarrow \chi_{B(x, r)} D_{i} f \text { in } L^{1}\left(\mathbb{R}^{n}\right) \text { as } k \rightarrow \infty .
$$

On the other hand, we get that

$$
\begin{aligned}
D_{i} M f(x) & \geq \lim _{k \rightarrow \infty} \frac{1}{h_{k}}\left(\underset{B\left(x+h_{k} e_{i}, r\right)}{f} f(y) d y-f_{B(x, r)} f(y) d y\right) \\
& =\lim _{k \rightarrow \infty} \frac{1}{m(B(x, r))} \int_{B(x, r)} \frac{f\left(y+h_{k} e_{i}\right)-f(y)}{h_{k}} d y=f_{B(x, r)} D_{i} f(y) d y .
\end{aligned}
$$

Suppose instead that $r=0$. The proof of the lower bound of $D_{i} M f(x)$ applies now, too, and we get that $D_{i} M f(x) \geq D_{i} f(x)$. If we have $r_{k}=0$ for infinitely many $k$, we can decide straightforwardly that $D_{i} M f(x)=D_{i} f(x)$. If $r_{k}>0$ starting from some $k_{0}$, we get by the same way as when studying the upper bound of $D_{i} M f(x)$ in the case $r>0$ that

$$
D_{i} M f(x) \leq \lim _{k \rightarrow \infty} f_{B\left(x, r_{k}\right)} f_{h_{k}}^{i}(y) d y=D_{i} f(x),
$$

because

$$
\begin{aligned}
& \lim _{k \rightarrow \infty}\left|f_{B\left(x, r_{k}\right)} f_{h_{k}}^{i}(y) d y-D_{i} f(x)\right|=\lim _{k \rightarrow \infty}\left|f_{B\left(x, r_{k}\right)}\left(f_{h_{k}}^{i}(y)-D_{i} f(y)\right) d y\right| \\
& \leq \lim _{k \rightarrow \infty} M\left(f_{h_{k}}^{i}-D_{i} f\right)(x)=0 .
\end{aligned}
$$

Now we have shown the claim in the ball $B(0, R)$. Since $R$ was arbitrary, this completes the proof.

\section{Continuity of the maximal operator in $W^{1, p}\left(\mathbb{R}^{n}\right)$}

By using Theorem 3.1 and Lemma 2.2, we can establish quite easily our main result which verifies the continuity of the maximal operator in $W^{1, p}\left(\mathbb{R}^{n}\right)$.

Theorem 4.1. $M: W^{1, p}\left(\mathbb{R}^{n}\right) \mapsto W^{1, p}\left(\mathbb{R}^{n}\right)$ is continuous for all $1<p<\infty$.

Proof. Let $f_{j} \rightarrow f$ in $W^{1, p}\left(\mathbb{R}^{n}\right)$ when $j \rightarrow \infty$. We have to show that $\left\|M f_{j}-M f\right\|_{1, p}$ $\rightarrow 0$. Because we know the continuity of $M$ in $L^{p}\left(\mathbb{R}^{n}\right)$, it is sufficient to prove that 
$\left\|D_{i} M f_{j}-D_{i} M f\right\|_{p} \rightarrow 0$ for all $i, 1 \leq i \leq n$. Also it is clear that we may assume the functions $f_{j}$ and $f$ to be nonnegative.

Let $\varepsilon>0$ be fixed but arbitrary. We start by choosing $R>0$ so that $\left\|2 M D_{i} f\right\|_{p, C_{1}}$ $<\varepsilon$, where $C_{1}=\mathbb{R}^{n} \backslash B(0, R)$. By absolute continuity we choose $\alpha>0$ so that $\left\|2 M D_{i} f\right\|_{p, A}<\varepsilon$ always when $m(A)<\alpha$ and $A$ is a measurable subset of $B(0, R)$.

We let (compare with the remark after Definition 2.1) $u_{x}(r)$ stand for the average of $D_{i} f$ in the ball $B(x, r)$ and $u_{x}(0)=D_{i} f(x)$. As already observed, for almost every $x \in \mathbb{R}^{n}$ the functions $u_{x}$ are continuous on $[0, \infty)$ and converge to 0 when $r \rightarrow \infty$. Consequently for almost every $x$ the function $u_{x}$ is uniformly continuous on $[0, \infty)$ and therefore we can find $\delta(x)>0$ such that $\left|u_{x}\left(r_{1}\right)-u_{x}\left(r_{2}\right)\right|<\frac{\varepsilon}{\left(m\left(B_{R}\right)\right)^{\frac{1}{p}}}$ when $\left|r_{1}-r_{2}\right|<\delta(x)$. Now we write that

$$
B_{R}=\left(\bigcup_{i=1}^{\infty}\left\{x \in B_{R}: \delta(x)>\frac{1}{i}\right\}\right) \cup \mathcal{N}
$$

where $m(\mathcal{N})=0$. From that we infer that there exists $\delta>0$ such that

$$
\begin{aligned}
& m\left(\left\{x \in B_{R}:\left|u_{x}\left(r_{1}\right)-u_{x}\left(r_{2}\right)\right|>\frac{\varepsilon}{\left(m\left(B_{R}\right)\right)^{\frac{1}{p}}} \text { for some } r_{1}, r_{2},\left|r_{1}-r_{2}\right|<\delta\right\}\right) \\
& =: m\left(C_{2}\right)<\frac{\alpha}{2} .
\end{aligned}
$$

The set $C_{2}$ is easily shown to be measurable. Furthermore, Lemma 2.2 says that we can find $j_{0}$ so that

$$
m\left(\left\{x: \mathcal{R} f_{j}(x) \not \subset \mathcal{R} f(x)_{(\delta)}\right\}\right)=: m\left(C^{j}\right)<\frac{\alpha}{2} \text { when } j \geq j_{0} .
$$

Then, let $j \geq j_{0}$ be fixed. It follows from Theorem 3.1 that almost everywhere in $\mathbb{R}^{n}$

$$
\begin{aligned}
& \left|D_{i} M f_{j}(x)-D_{i} M f(x)\right|=\left|\underset{B\left(x, r_{1}\right)}{f} D_{i} f_{j}(y) d y-f_{B\left(x, r_{2}\right)} D_{i} f(y) d y\right| \\
\leq & \left|f_{B\left(x, r_{1}\right)} D_{i} f_{j}(y) d y-f_{B\left(x, r_{1}\right)} D_{i} f(y) d y\right| \\
& +\left|f_{B\left(x, r_{1}\right)} D_{i} f(y) d y-f_{B\left(x, r_{2}\right)} D_{i} f(y) d y\right| \\
\leq & M\left(D_{i} f_{j}-D_{i} f\right)(x)+\left|f_{B\left(x, r_{1}\right)} D_{i} f(y) d y-f_{B\left(x, r_{2}\right)} D_{i} f(y) d y\right|
\end{aligned}
$$

for all $r_{1} \in \mathcal{R} f_{j}(x), r_{2} \in \mathcal{R} f(x)$. This inequality applies also to the cases $r_{1}=0$ or $r_{2}=0$ when we agree that

$$
f_{B(x, 0)} D_{i} f(y) d y:=D_{i} f(x) .
$$

This is obvious because for almost every $x$ it is true that $M f(x) \geq f(x)$, and by Theorem $3.1 D_{i} M f(x)=D_{i} f(x)$ if $0 \in \mathcal{R} f(x)$. 
Now, if $x \notin C_{1} \cup C_{2} \cup C^{j}$, we can pick $r_{1} \in \mathcal{R} f_{j}(x)$ and $r_{2} \in \mathcal{R} f(x)$ so that $\left|r_{1}-r_{2}\right|<\delta$. Our choice of $\delta$ implies that

$$
s:=\left|f_{B\left(x, r_{1}\right)} D_{i} f(y) d y-f_{B\left(x, r_{2}\right)} D_{i} f(y) d y\right|<\frac{\varepsilon}{\left(m\left(B_{R}\right)\right)^{\frac{1}{p}}} .
$$

If $x \in C_{1} \cup C_{2} \cup C^{j}$, we estimate that $s \leq 2 M D_{i} f(x)$. Observe also that $m\left(C_{2} \cup C^{j}\right)<\alpha$.

Combining the above estimates it follows that

$$
\begin{aligned}
\left\|D_{i} M f_{j}-D_{i} M f\right\|_{p, \mathbb{R}^{n}} \leq & \left\|M\left(D_{i} f_{j}-D_{i} f\right)\right\|_{p, \mathbb{R}^{n}}+\left\|\frac{\varepsilon}{\left(m\left(B_{R}\right)\right)^{\frac{1}{p}}}\right\|_{p, B_{R}} \\
& +\left\|2 M D_{i} f\right\|_{p, C_{1}}+\left\|2 M D_{i} f\right\|_{p, C_{2} \cup C_{j}} .
\end{aligned}
$$

The first term in the right-hand side of the inequality converges to zero when $j \rightarrow \infty$. The rest of the terms are less than $\varepsilon$, because of the choices of $R$ and $\alpha$. As $\varepsilon$ was arbitrary we conclude that $\left\|D_{i} M f_{j}-D_{i} M f\right\|_{p} \rightarrow 0$ as $j \rightarrow \infty$. The proof is complete.

Remark. One may ask, what kind of estimates we can find for the modulus of continuity of $M$. Quite surprisingly, it turns out that there does not exist a function $F:(0, \infty) \mapsto(0, \infty)$ such that

$$
\|M f-M g\|_{1, p} \leq F\left(\|f-g\|_{1, p}\right) \text { for all } f, g \in W^{1, p}\left(\mathbb{R}^{n}\right) .
$$

This is a consequence of the following two facts. First, $M$ is not Lipschitz-continuous in $W^{1, p}\left(\mathbb{R}^{n}\right)$, because this would imply that $M$ is bounded in $W^{2, p}\left(\mathbb{R}^{n}\right)$ which is not true (see for example $[\mathrm{Ko}]$ ). The philosophy of this phenomenon is that even the maximal function of a smooth positive function usually has angles in its graph. Second, the maximal operator is scale-invariant, thus $M(c f)=c M f$ for all $c>0$. We thank Jani Onninen for pointing out the first fact.

\section{ACKNOWLEDGEMENTS}

I would like to thank Eero Saksman for the advice and support he has given me. I also thank Juha Kinnunen and Jani Onninen for their valuable comments on the manuscript.

\section{REFERENCES}

[AL] F.J. Almgren and E.H. Lieb, Symmetric decreasing rearrangement is sometimes continuous, J. Amer. Math. Soc. 2 (1989), 683-773. MR.1002633 (90f:49038)

[GT] D. Gilbarg and N.S. Trudinger, Elliptic Partial Differential Equations of Second Order, 2nd ed., Springer-Verlag, Berlin, 1983. MR0737190 (86c:35035)

[HO] P. Hajłasz and J. Onninen, On boundedness of maximal functions in Sobolev spaces. Ann. Acad. Sci. Fenn. Math. 29 (2004), 167-176. MR2041705 (2005a:42010)

[K] J. Kinnunen, The Hardy-Littlewood maximal function of a Sobolev-function, Israel J. Math. 100 (1997), 117-124. MR.1469106 (99a:30029)

[KL] J. Kinnunen and P. Lindqvist, The derivative of the maximal function, J. Reine Angew. Math. 503 (1998), 161-167. MR1650343 (99j:42027) 
[KS] J. Kinnunen and E. Saksman, Regularity of the fractional maximal function, Bull. London Math. Soc. 35 (2003), no. 4, 529-535. MR.1979008 (2004e:42035)

[Ko] S. Korry, Boundedness of Hardy-Littlewood maximal operator in the framework of LizorkinTriebel spaces, Rev. Mat. Complut. 15 (2002), 401-416. MR1951818 (2004a:42020)

Department of Mathematics and Statistics, University of Jyväskylä, P.O. Box 35 (MAD), 40014 University of JyvÄSKYlä, Finland

E-mail address: haluiro@maths.jyu.fi 